\title{
Regularity for Suitable Weak Solutions to the Navier-Stokes Equations in Critical Morrey Spaces
}

\author{
G. Seregin
}

\begin{abstract}
A class of sufficient conditions of local regularity for suitable weak solutions to the nonstationary three-dimensional Navier-Stokes equations are discussed. The corresponding results are formulated in terms of functionals which are invariant with respect to the Navier-Stokes equations scaling. The famous Caffarelli-Kohn-Nirenberg condition is contained in that class as a particular case.
\end{abstract}

1991 Mathematical subject classification (Amer. Math. Soc.): 35K, $76 \mathrm{D}$.

Key Words: Navier-Stokes equations, suitable weak solutions, local regularity theory.

\section{Introduction and Main Result}

In the present paper, we address the problem of smoothness of a certain class of weak solutions to the nonstationary three-dimensional Navier-Stokes equations (NSE's)

$$
\partial_{t} v+v \cdot \nabla v-\Delta v=-\nabla p, \quad \operatorname{div} v=0 .
$$

In our setting, they are considered in the unit space-time cylinder $Q=B \times]-$ 1,0 [, where $B$ is the unit ball of $\mathbb{R}^{3}$ centered at the origin. As usual, $v$ and $p$ stand for the velocity field and for the pressure field, respectively. We ask the following question. What are minimal conditions which ensure regularity of the velocity field $v$ at the space-time origin $z=(x, t)=(0,0)=0$ ? Our definition of regularity of $v$ at the point $z=0$ means that there exists a number $r \in] 0,1$ ] such that $v$ is a Hölder continuous function in the completion of the space-time cylinder $Q(r)=B(r) \times]-r^{2}, 0[$. Here, $B(r)$ is the ball of radius $r$ centered at the origin so that $B=B(1)$. This definition is due to 
O. Ladyzhenskaya and the author, see [5], and slightly differs from the most popular one by L. Caffarelli, R.-V. Kohn, and L. Nirenberg given in their celebrated paper [1].

Our interest to the above question is motivated by the important observation of J. Leray made in his remarkable paper [7]. J. Leray proved the uniqueness of regular solutions in the class of turbulent solutions which are also called weak Leray-Hopf solutions. For exact definitions, we refer the reader to the paper [7] and, for example, to papers [5] and [10].

We restrict ourselves to the analysis of the so-called suitable weak solutions also introduced in [1]. Here, we follow the definition of suitable weak solution in F.-H. Lin's reduction, see $[8]$ and also $[5]$ for discussions on other definitions.

Definition 1.1 We say that the pair $v$ and $p$ is a suitable weak solution to the NSE's in $Q$ if the following conditions are fulfilled:

$$
v \in L_{2, \infty}(Q) \cap W_{2}^{1,0}(Q), \quad p \in L_{\frac{3}{2}}(Q) ;
$$

the NSE's hold in $Q$ in the sense of distributions;

for a.a. $t \in]-1,0[$, the local energy inequality

$$
\begin{aligned}
\int_{B} \varphi(x, t)|v(x, t)|^{2} d x & +2 \int_{-1}^{t} \int_{B} \varphi|\nabla v|^{2} d x d t^{\prime} \leq \int_{-1}^{t} \int_{B}\left(|v|^{2}\left(\Delta \varphi+\partial_{t} \varphi\right)\right. \\
& \left.+v \cdot \varphi\left(|v|^{2}+2 p\right)\right) d x d t^{\prime}
\end{aligned}
$$

holds for all non-negative functions $\varphi \in C_{0}^{\infty}\left(\mathbb{R}^{3} \times \mathbb{R}^{1}\right)$ vanishing in a neighborhood of the parabolic boundary $\partial^{\prime} Q$ of the cylinder $Q$.

Here, the following notion has been used:

$$
L_{m, n}(Q)=L_{n}\left(-1,0 ; L_{m}(B)\right), \quad W_{2}^{1,0}(Q)=L_{2}\left(-1,0 ; W_{2}^{1}(B)\right),
$$

and $L_{m}(B)$ and $W_{m}^{1}(B)$ are the usual Lebesgue and Sobolev spaces, respectively.

Let us comment Definition 1.1 briefly. The most essential part of it is local energy inequality (1.4). Unfortunately, we do not know whether or not any weak Leray-Hopf solution to the initial boundary value problem for the 
NSE's satisfies the local energy inequality but at least one of them does so. Moreover, in the case of local (in time) strong solvability of that problem, its solution belongs to the class of suitable weak solutions at least up to the moment of time when the first singularity occurs. Our assumption on the pressure field $p$, see (1.2), is motivated by the linear theory. In this sense, it is valid for any weak Leray-Hopf solution if the data of the initial boundary value problem are not too bad. Moreover, we may vary classes for the pressure filed. The space $L_{\frac{3}{2}}$ seems to be the most convenient for treating. For more details, we refer the reader to the paper [5].

It is known that system (1.1) is invariant with respect to the scaling

$$
v^{\lambda}(x, t)=\lambda v\left(\lambda x, \lambda^{2} t\right), \quad p^{\lambda}(x, t)=\lambda^{2} p\left(\lambda x, \lambda^{2} t\right) .
$$

We call this scaling the natural one.

In the local regularity theory, functionals being invariant to the natural scaling play a very important role. Here, it the list of some of them:

$$
\begin{gathered}
A(r)=\operatorname{ess} \sup _{-r^{2}<t<0} \frac{1}{r} \int_{B(r)}|v(x, t)|^{2} d x, \quad E(r)=\frac{1}{r} \int_{Q(r)}|\nabla v|^{2} d z, \\
C(r)=\frac{1}{r^{2}} \int_{Q(r)}|v|^{3} d z, \quad H(r)=\frac{1}{r^{3}} \int_{Q(r)}|v|^{2} d z, \\
D_{0}(r)=\frac{1}{r^{2}} \int_{Q(r)}\left|p-[p]_{B(r)}\right|^{\frac{3}{2}} d z,
\end{gathered}
$$

where

$$
[p]_{B(r)}=\frac{1}{|B(r)|} \int_{B(r)} p(x, t) d x .
$$

These functionals may be used to produce norms of special Morrey classes. We call them critical Morrey spaces. The list of scaling invariant functionals can be extended. For example, the norm $M_{s, l}(r)=\|v\|_{L_{s, l}(Q(r))}$ is invariant with respect to the natural scaling if $3 / s+2 / l=1$.

The most of results in the local regularity theory is formulated with the help of those functionals and have the form of the so-called $\varepsilon$-regularity conditions. A typical $\varepsilon$-regularity condition reads: if the norm of the velocity field $v$ in critical Morrey's space is small enough, then the space-time origin 
is a regular point of $v$. This is not completely rigorous statement but reflects the spirit of the $\varepsilon$-regularity theory quite well. For example, the famous Caffarelli-Kohn-Nirenberg condition can be formulated as follows.

Theorem 1.2 Let the pair $v$ and $p$ be a suitable weak solution to the NSE's in $Q$. There is a universal constant $\varepsilon_{0}$ such that if

$$
\sup _{0<r \leq 1} E(r)<\varepsilon_{0}
$$

then $z=0$ is a regular point of $v$.

Another important example is the local version of the Ladyzheskaya-ProdiSerrin condition (LPS-condition) proved by Serrin and Struwe in papers [13] and [14. In our situation, it reads

Theorem 1.3 Let the pair $v$ and $p$ be a suitable weak solution to the NSE's in $Q$. Assume that numbers $s$ and $l$ satisfy conditions

$$
\frac{3}{s}+\frac{2}{l}=1, \quad 3 \leq s \leq+\infty, \quad 2 \leq l \leq+\infty .
$$

There is a positive constant $\bar{\varepsilon}_{0}$ depending on $s$ and $l$ only such that if

$$
M_{s, l}(1)=\sup _{0<r \leq 1} M_{r, l}(r)<\bar{\varepsilon}_{0}
$$

then $z=0$ is a regular point of $v$.

Other examples can be founded, for instance, in the paper [10].

If we believe that suitable weak solutions are smooth, then it would be natural to get rid of smallness of scaling invariant functionals and show that their boundedness is sufficient for regularity. In a number of cases, it is obvious. For example, in the case of LPS-condition, if $s>3$, boundedness of $M_{s, l}(1)$, together with the absolute continuity of Lebesgue's integral and the natural scaling, allows us to assume that $M_{s, l}(1)$ is small as we wish. In the marginal case $s=3$ and $l=+\infty$, the above mentioned reduction is much more subtle and based on backward uniqueness results for the heat operator with variable lower order terms, see [12] and [3]. For functionals $A(r), C(r)$, and $E(r)$, this is an open problem, i.e., it is unknown whether or not their boundedness implies regularity. 
To formulate our main result, let us introduce the additional notation

$$
\begin{gathered}
G=\inf \left\{\limsup _{r \rightarrow 0} E(r), \limsup _{r \rightarrow 0} C(r), \limsup _{r \rightarrow 0} A(r)\right\}, \\
g=\inf \left\{\liminf _{r \rightarrow 0} E(r), \liminf _{r \rightarrow 0} C(r), \liminf _{r \rightarrow 0} A(r), \liminf _{r \rightarrow 0} H(r), \liminf _{r \rightarrow 0} D_{0}(r)\right\} .
\end{gathered}
$$

The main result of the paper is

Theorem 1.4 Assume that the pair $v$ and $p$ is a suitable weak solution to the NSE's in $Q$. For any $M>0$, there exists a positive number $\varepsilon(M)$ with the following property. If $G<M$ and $g<\varepsilon(M)$, then $z=0$ is a regular point of $v$.

Let us discuss simple consequences of Theorem 1.4. In particular, one has the following generalization of Theorem 1.2 .

Corollary 1.5 Assume that the pair $v$ and $p$ is a suitable weak solution to the NSE's in $Q$. Given $M>0$, let $\varepsilon(M)$ be the number of Theorem 1.4. If, for some $M>0$, two conditions $\lim \sup _{r \rightarrow 0} E(r)<M$ and $\liminf _{r \rightarrow 0} E(r)<$ $\varepsilon(M)$ hold, then $z=0$ is a regular point of $v$.

To see that Theorem 1.2 can be deduced from Corollary 1.5. it is sufficient to let $M=1$ and $\varepsilon_{0}=\min \{1, \varepsilon(1)\}$. Another direct consequence of Theorem 1.4 can be stated as follows.

Corollary 1.6 Assume that the pair $v$ and $p$ is a suitable weak solution to the NSE's in $Q$. If $G<+\infty$ and $g=0$, then $z=0$ is a regular point of $v$.

Certainly, there are other versions of Theorem 1.4. For example, we have the following statement.

Theorem 1.7 Assume that the pair $v$ and $p$ is a suitable weak solution to the NSE's in $Q$. For any $M>0$, there exists a positive number $\widehat{\varepsilon}(M)$ with the following property. Let $G<M$ and

$$
\liminf _{r \rightarrow 0} E_{3}(r)<\widehat{\varepsilon}(M),
$$

where

$$
E_{3}(r)=\frac{1}{r} \int_{Q(r)}\left|v_{, 3}\right|^{2} d z, \quad v_{, 3}=\frac{\partial v}{\partial x_{3}} .
$$

Then $z=0$ is a regular point of $v$.

Acknowledgement The work was supported by the Alexander von Humboldt Foundation, by the RFFI grant 05-01-00941-a, and by the CRDF grant RU-M1-2596-ST-04. 


\section{Estimates of Suitable Weak Solutions to the NSE's}

In this section, we would like to present the list of the main estimates of suitable weak solutions to the NSE's in $Q$. The first of them is a consequence of multiplicative inequalities and has the form

$$
C(r) \leq c\left[\left(\frac{\varrho}{r}\right)^{3} A^{\frac{3}{4}}(\varrho) E^{\frac{3}{4}}(\varrho)+\left(\frac{r}{\varrho}\right)^{3} A^{\frac{3}{2}}(\varrho)\right]
$$

for all $0<r \leq \varrho \leq 1$. Here and in what follows, we denote all positive universal constants by $c$. A proof of (2.1) is given, for example, in [5].

There are two consequences of the local energy inequality:

$$
A(R / 2)+E(R / 2) \leq c\left[C^{\frac{2}{3}}(R)+C(R)+C^{\frac{1}{3}}(R) D_{0}^{\frac{2}{3}}(R)\right]
$$

and

$$
A(R / 2)+E(R / 2) \leq c\left[C^{\frac{2}{3}}(R)+C^{\frac{1}{3}}(R) D_{0}^{\frac{2}{3}}(R)+A^{\frac{1}{2}}(R) C^{\frac{2}{3}}(R) E^{\frac{1}{2}}(R)\right]
$$

for all $0<R \leq 1$. Inequality (2.2) follows from the local energy inequality directly, a proof of (2.3) can be found in [5].

There are also three versions of the decay estimate for the pressure:

$$
D_{0}(r) \leq c\left[\left(\frac{r}{\varrho}\right)^{\frac{5}{2}} D_{0}(\varrho)+\left(\frac{\varrho}{r}\right)^{2} C(\varrho)\right]
$$

or

$$
D_{0}(r) \leq c\left[\left(\frac{r}{\varrho}\right)^{\frac{5}{2}} D_{0}(\varrho)+\left(\frac{\varrho}{r}\right)^{2} A^{\frac{1}{2}}(\varrho) E(\varrho)\right]
$$

or

$$
D_{0}(r) \leq c\left[\left(\frac{r}{\varrho}\right)^{\frac{5}{2}} D_{0}(\varrho)+\left(\frac{\varrho}{r}\right)^{3} A^{\frac{3}{4}}(\varrho) E^{\frac{3}{4}}(\varrho)\right] .
$$

Inequality (2.4)-(2.6) are valid for all $0<r \leq \varrho \leq 1$. Inequality (2.4) is proved in [9] and inequalities (2.5) and (2.6) are discussed in [11].

The following lemma shows that if one of the quantities $\sup _{m<r \leq 1} E(r)$, $\sup _{m<r \leq 1} C(r)$, or $\sup _{m<r \leq 1} A(r)$ is bounded, then all others are bounded too. 
Lemma 2.1 Suppose that the pair $v$ and $p$ is a suitable weak solution to the Navier-Stokes equations in $Q$. The following estimates are valid:

a) Let

$$
\sup _{0<r \leq 1} E(r)=E_{0}<+\infty .
$$

Then there exists a positive constant d depending only on $E_{0}$ such that

$$
A^{\frac{3}{2}}(r)+C(r)+D_{0}^{2}(r) \leq d\left(E_{0}\right)\left(r^{\frac{1}{2}}\left(A^{\frac{3}{2}}(1)+D_{0}^{2}(1)\right)+1\right)
$$

for all $0<r \leq 1 / 4$;

b) Let

$$
\sup _{0<r \leq 1} C(r)=C_{0}<+\infty
$$

Then

$$
A(r)+D_{0}(r)+E(r) \leq c\left(r^{2} D_{0}(1)+C_{0}+C_{0}^{\frac{2}{3}}\right)
$$

for all $0<r \leq 1 / 2$;

c) Let

$$
\sup _{0<r \leq 1} A(r)=A_{0}<+\infty .
$$

Then there exists a positive constant e depending only on $A_{0}$ such that

$$
C^{\frac{4}{3}}(r)+D_{0}(r)+E(r) \leq e\left(A_{0}\right)\left(r^{2}\left(D_{0}(1)+E(1)\right)+1\right)
$$

for all $0<r \leq 1 / 2$.

A proof Lemma 2.1 is based upon estimates (2.1)-(2.6) and presented in [11].

\section{Proof of Theorem 1.4}

The key part of the proof of Theorem 1.4 is

Proposition 3.1 Let the pair $v$ and $p$ be a suitable weak solution to the $N S E$ 's in $Q$.

For any $M>0$, there exists a positive number $\varepsilon_{10}=\varepsilon_{10}(M)$ with the following property. If

$$
\sup _{0<r \leq 1} E(r)=E_{0} \leq M
$$


and

$$
g_{r_{*}}=\min \left\{E\left(r_{*}\right), A\left(r_{*}\right), C\left(r_{*}\right), H\left(r_{*}\right), D_{0}\left(r_{*}\right)\right\}<\varepsilon_{10}(M)
$$

for some $\left.r_{*} \in\right] 0, \min \left\{1 / 4,\left(A^{\frac{3}{2}}(1)+D_{0}^{2}(1)\right)^{-2}\right\}[$, then $z=0$ is a regular point of $v$.

Proof Assume that the statement of the proposition is false. Then there exist a positive number $M$ and a sequence of suitable weak solutions $v^{n}$ and $p^{n}$ to the NSE's in $Q$ such that, for any $n \in \mathbb{N}$, the following two conditions hold

$$
E\left(v^{n}, r\right)=\frac{1}{r} \int_{Q(r)}\left|\nabla v^{n}\right|^{2} d z \leq M
$$

for all $0<r \leq 1$ and

$$
\begin{gathered}
g_{r_{n}}\left(v^{n}, p^{n}\right)= \\
=\min \left\{E\left(v^{n}, r_{n}\right), A\left(v^{n}, r_{n}\right), C\left(v^{n}, r_{n}\right), H\left(v^{n}, r_{n}\right), D_{0}\left(p^{n}, r_{n}\right)\right\} \leq \frac{1}{n}
\end{gathered}
$$

for some

$$
\left.r_{n} \in\right] 0, \min \left\{1 / 4,\left(A^{\frac{3}{2}}\left(v^{n}, 1\right)+D_{0}^{2}\left(p^{n}, 1\right)\right)^{-2}\right\}[,
$$

but $z=0$ is a singular point of $v^{n}$. Here, we have used the notation

$$
\begin{aligned}
& A\left(v^{n}, r\right)=\operatorname{ess} \sup _{-r^{2}<t<0} \frac{1}{r} \int_{B(r)}|v(x, t)|^{2} d x, \quad C\left(v^{n}, r\right)=\frac{1}{r^{2}} \int_{Q(r)}\left|v^{n}\right|^{3} d z, \\
& H\left(v^{n}, r\right)=\frac{1}{r^{3}} \int_{Q(r)}\left|v^{n}\right|^{2} d z, \quad D_{0}\left(p^{n}, r\right)=\frac{1}{r^{2}} \int_{Q(r)}\left|p^{n}-\left[p^{n}\right]_{B(r)}\right|^{\frac{3}{2}} d z .
\end{aligned}
$$

On the other hand, since $z=0$ is a singular point of $v^{n}$, there exists a universal positive number $\varepsilon$ such that

$$
C\left(v^{n}, r\right)+D_{0}\left(p^{n}, r\right) \geq \varepsilon>0
$$

for all $0<r \leq 1$, see, for example, [5. We emphasize that (3.6) is valid for any natural number $n$.

By Lemma 2.1] and by the properties of $r_{n}$, see (3.5), we find the estimate

$$
A^{\frac{3}{2}}\left(v^{n}, r\right)+C\left(v^{n}, r\right)+D_{0}^{2}\left(p^{n}, r\right) \leq
$$




$$
\leq d(M)\left[\left(\frac{r}{r_{n}}\right)^{\frac{1}{2}} r_{n}^{\frac{1}{2}}\left(A^{\frac{3}{2}}\left(v^{n}, 1\right)+D_{0}^{2}\left(p^{n}, 1\right)\right)+1\right] \leq d_{0}(M)
$$

for all $r \in] 0, r_{n}[$.

Now, let us scale our functions $v^{n}$ and $p^{n}$ so that

$$
u^{n}(y, s)=r_{n} v^{n}\left(r_{n} y, r_{n}^{2} s\right), \quad q^{n}(y, s)=r_{n}^{2} p^{n}\left(r_{n} y, r_{n}^{2} s\right)
$$

for $(y, s) \in Q$. By the invariance of functionals and equations with respect to the natural scaling, we have:

the pair $u^{n}$ and $q^{n}$ is a suitable weak solution to the NSE's in $Q$

for each $n \in \mathbb{N}$;

$$
E\left(u^{n}, r\right) \leq M
$$

for all $0<r \leq 1$ and for each $n \in \mathbb{N}$;

$$
\begin{gathered}
g_{r_{n}}\left(v^{n}, p^{n}\right)=g_{1}\left(u^{n}, q^{n}\right) \rightarrow 0 \quad \text { as } \quad n \rightarrow+\infty ; \\
C\left(u^{n}, r\right)+D_{0}\left(q^{n}, r\right) \geq \varepsilon>0
\end{gathered}
$$

for all $0<r \leq 1$ and for each $n \in \mathbb{N}$;

$$
A^{\frac{3}{2}}\left(u^{n}, r\right)+C\left(u^{n}, r\right)+D_{0}^{2}\left(q^{n}, r\right) \leq d_{0}(M)
$$

for all $0<r \leq 1$ and for each $n \in \mathbb{N}$.

Now, let $n$ tend to $+\infty$. First of all, in order to pass to the limit in non-linear terms, a strong compactness is needed. To this end, we estimate the weak derivative of $v$ in $t$ using the equation in the standard way:

$$
\begin{gathered}
\int_{Q} \partial_{t} u^{n} \cdot w d z=\int_{Q}\left(u^{n} \otimes u^{n}: \nabla w-\nabla u^{n}: \nabla w+\left(q^{n}-\left[q^{n}\right]_{B}\right) \operatorname{div} w\right) d z \leq \\
\leq\left(\int_{Q}\left|u^{n}\right|^{3} d z\right)^{\frac{2}{3}}\left(\int_{Q}|\nabla w|^{3} d z\right)^{\frac{1}{3}}+\left(\int_{Q}\left|\nabla u^{n}\right|^{2} d z\right)^{\frac{1}{2}}\left(\int_{Q}|\nabla w|^{2} d z\right)^{\frac{1}{2}}+ \\
+\left(\int_{Q}\left|q^{n}-\left[q^{n}\right]\right|^{\frac{3}{2}} d z\right)^{\frac{2}{3}}\left(\int_{Q}|\operatorname{div} w|^{3} d z\right)^{\frac{1}{3}} \leq \\
\leq c\left(C^{\frac{2}{3}}\left(u^{n}, 1\right)+D_{0}^{\frac{2}{3}}\left(q^{n}, 1\right)+E^{\frac{1}{2}}\left(u^{n}, 1\right)\right)\left(\int_{Q}|\nabla w|^{3} d z\right)^{\frac{1}{3}} \leq
\end{gathered}
$$




$$
\leq d_{1}(M)\left(\int_{Q}|\nabla w|^{3} d z\right)^{\frac{1}{3}} .
$$

The latter estimate holds for any $w \in C_{0}^{\infty}(Q)$. By the density arguments, it is valid for any $w \in L_{3}\left(-1,0 ; \stackrel{\circ}{W}_{3}^{1}(B)\right)$. So, we have

$$
\left\|\partial_{t} u^{n}\right\|_{L_{3 / 2}\left(-1,0 ; W_{3 / 2}^{-1}(B)\right)} \leq d_{1}(M) .
$$

The final estimate comes from the known multiplicative inequality and has the form

$$
\int_{Q}\left|u^{n}\right|^{\frac{10}{3}} d z \leq d_{2}(M) .
$$

Now, using known compactness arguments and selecting a subsequence if necessary, we find

$$
\begin{aligned}
& u^{n} \stackrel{\star}{\rightarrow} u \quad \text { in } \quad L_{2, \infty}(Q), \\
& \nabla u^{n} \rightarrow \nabla u \quad \text { in } \quad L_{2}(Q) \text {, } \\
& u^{n} \rightarrow u \quad \text { in } \quad L_{3}(Q), \\
& q^{n}-\left[q^{n}\right]_{B} \rightarrow q \text { in } \quad L_{\frac{3}{2}}(Q)
\end{aligned}
$$

Moreover, the pair $u$ and $q$ ia a suitable weak solution to the NSE's in $Q$ and

$$
A^{\frac{3}{2}}(u, r)+C(u, r)+D_{0}^{2}(q, r) \leq d_{0}(M)
$$

for all $0<r \leq 1$.

Now, let us see what follows from (3.10). It is easy to observe that there exists a subsequence $\left\{n_{k}\right\}_{k=1}^{\infty}$ such that either

$$
E\left(u^{n_{k}}, 1\right) \rightarrow 0
$$

or

$$
C\left(u^{n_{k}}, 1\right) \rightarrow 0,
$$

or

$$
A\left(u^{n_{k}}, 1\right) \rightarrow 0
$$

or

$$
H\left(u^{n_{k}}, 1\right) \rightarrow 0
$$


or

$$
D_{0}\left(q^{n_{k}}, 1\right) \rightarrow 0
$$

as $k \rightarrow+\infty$.

Let us discuss each case separately, starting with (3.17). According to (3.17), we have

$$
\nabla u^{n_{k}} \rightarrow 0 \quad \text { in } \quad L_{2}(Q)
$$

and $\nabla u=0$ in $Q$. Therefore, $E(u, r)=0$ for all $r \in] 0,1]$ and, by Theorem 1.2. $z=0$ is a regular point of $u$ and, in particular, there exists a number $0<r_{1} \leq 1$ such that

$$
\sup _{z \in \bar{Q}\left(r_{1}\right)}|u(z)| \leq d_{3}(M)
$$

Passing to the limit in (3.11), we show

$$
C(u, r)+\limsup _{k \rightarrow \infty} D_{0}\left(q^{n_{k}}, r\right) \geq \varepsilon
$$

for all $0<r \leq 1$. Thanks to (3.23), it follows from (3.24) that

$$
c d_{3}^{3}(M) r^{3}+\limsup _{k \rightarrow \infty} D_{0}\left(q^{n_{k}}, r\right) \geq \varepsilon
$$

for all $0<r \leq r_{1}$.

Functions $u^{n_{k}}$ and $q^{n_{k}}$ satisfy decay estimate (2.5). So, according to (3.12), we have

$$
\begin{gathered}
D_{0}\left(q^{n_{k}}, r\right) \leq c\left[\left(\frac{r}{1}\right)^{\frac{5}{2}} D_{0}\left(q^{n_{k}}, 1\right)+\left(\frac{1}{r}\right)^{2} A^{\frac{1}{2}}\left(u^{n_{k}}, 1\right) E\left(u^{n_{k}}, 1\right)\right] \\
\leq d_{4}(M)\left[r^{\frac{5}{2}}+\left(\frac{1}{r}\right)^{2} E\left(u^{n_{k}}, 1\right)\right]
\end{gathered}
$$

for all $0<r \leq 1$. It remains to take the limit as $k \rightarrow \infty$ and, by (3.22), arrive at the inequality

$$
\limsup _{k \rightarrow \infty} D_{0}\left(q^{n_{k}}, r\right) \leq d_{4}(M) r^{\frac{5}{2}}
$$

for all $0<r \leq 1$. So, the latter estimate, together with (3.25), gives us the relation

$$
c d_{3}^{3}(M) r^{3}+d_{4}(M) r^{\frac{5}{2}} \geq \varepsilon
$$

for all $0<r \leq r_{1}$. It is not true for sufficiently small $r$. So, possibility of (3.17) is excluded. 
Now, assume that (3.18) takes place. Here, we are going to use inequality (3.12) and decay estimate (2.4). They lead us to the estimate

$$
\begin{gathered}
D_{0}\left(q^{n_{k}}, r\right) \leq c\left[r^{\frac{5}{2}} D_{0}\left(q^{n_{k}}, 1\right)+\frac{1}{r^{2}} C\left(u^{n_{k}}, 1\right)\right] \\
\leq d_{5}(M)\left[r^{\frac{5}{2}}+\frac{1}{r^{2}} C\left(u^{n_{k}}, 1\right)\right]
\end{gathered}
$$

for all $0<r \leq 1$. But it follows from (3.11) that

$$
\left.\left.\varepsilon \leq d_{5}(M)\left[r^{\frac{5}{2}}+\frac{1}{r^{2}} C\left(u^{n_{k}}, 1\right)\right]+C\left(u^{n_{k}}, r\right), \quad r \in\right] 0,1\right] .
$$

Taking into account (3.18) and passing to the limit in (3.27) as $k \rightarrow+\infty$, we show

$$
\left.\left.\varepsilon \leq d_{5}(M) r^{\frac{5}{2}}, \quad r \in\right] 0,1\right],
$$

which is also wrong for sufficiently small $r$. So, case (3.18) is excluded as well.

Cases (3.19) and (3.20) can be reduced to the previous one. Indeed, in both cases the limit equation is $u=0$. So, $C\left(u^{n_{k}}, r\right) \rightarrow C(u, r)=0$ as $k \rightarrow+\infty$ for all $0<r \leq 1$. Repeating estimates (3.26) and (3.27), we see that cases (3.19) and (3.20) do not occur either.

It remains to consider case (3.21). Here, we have $q^{n_{k}}-\left[q^{n_{k}}\right] \rightarrow 0$ in $L_{\frac{3}{2}}(Q)$ as $k \rightarrow+\infty$. Since

$$
0 \leq D_{0}\left(q^{n_{k}}, r\right) \leq \frac{c}{r^{2}} D_{0}\left(q^{n_{k}}, 1\right) \rightarrow 0
$$

we find

$$
C(u, r) \geq \varepsilon>0
$$

for all $0<r \leq 1$. Let us describe the properties of the limit function $u$ :

$$
u \in L_{2, \infty}(Q) \cap W_{2}^{1,0}(Q)
$$

the function $u$ satisfies the system of equations

$$
\partial_{t} u+u \cdot \nabla u-\Delta u=0, \quad \operatorname{div} u=0
$$

in $Q$ in the sense of distributions; 
for a.a. $t \in]-1,0[$, the function $u$ satisfies the local energy inequality

$$
\begin{aligned}
& \int_{B} \varphi(x, t)|u(x, t)|^{2} d x+2 \int_{-1}^{t} \int_{B} \varphi|\nabla u|^{2} d x d t^{\prime} \leq \\
& \leq \int_{-1}^{t} \int_{B}\left(|u|^{2}\left(\Delta \varphi+\partial_{t} \varphi\right)+|u|^{2} u \cdot \nabla \varphi\right) d x d t^{\prime}
\end{aligned}
$$

for all non-negative functions $\varphi \in C_{0}^{\infty}\left(\mathbb{R}^{3} \times \mathbb{R}\right)$ vanishing in a neighborhood of the parabolic boundary of the cylinder $Q$.

As it is shown in the Appendix, the function $u$, enjoying properties (3.29)(3.31), is, in fact, smoother. More precisely, $u$ is Hölder continuous say in $\bar{Q}(1 / 4)$ and, in particular,

$$
\sup _{z \in \bar{Q}(1 / 4)}|u(z)| \leq d_{6}(M)
$$

and, from (3.28), it follows that

$$
c d_{6}^{3}(M) r^{3} \geq \varepsilon
$$

for all $0<r \leq 1 / 4$, which is also not true. So, case (3.21) is excluded. Since our observations contradict with (3.10), we may conclude that the statement of Proposition 3.1 is valid. Proposition 3.1 is proved.

Proposition 3.2 Let the pair $v$ and $p$ be a suitable weak solution to the $N S E$ 's in $Q$. If

$$
\limsup _{r \rightarrow 0} E(r)<\frac{1}{2} m=M
$$

and

$$
g<\frac{1}{2} \varepsilon_{10}(m)=\varepsilon_{1}(M),
$$

then $z=0$ is a regular point of $v$.

Proof By condition (3.32), we can find a number $\left.\left.r_{1} \in\right] 0,1\right]$ such that

$$
\sup _{0<r \leq r_{1}} E(r) \leq m
$$


and scale $v$ and $p$ so that

$u(x, t)=r_{1} v\left(r_{1} x, r_{1}^{2} t\right), \quad q(x, t)=r_{1}^{2} p\left(r_{1} x, r_{1}^{2} t\right), \quad|x|<1, \quad-1<t<0$.

The pair $u$ and $q$ is then a suitable weak solution to the NSE's in $Q$ and

$$
\sup _{0<r \leq 1} E(u, r) \leq m
$$

and

$$
g(u, q)<\frac{1}{2} \varepsilon_{10}(m) .
$$

By (3.34), among quantities $\liminf _{r \rightarrow 0} E(u, r), \liminf _{r \rightarrow 0} A(u, r), \liminf _{r \rightarrow 0}$ $C(u, r), \liminf _{r \rightarrow 0} H(u, r)$, and $\liminf _{r \rightarrow 0} D_{0}(q, r)$, there should be at least one, which is less than $1 / 2 \varepsilon_{10}(m)$. For example, let $\liminf _{r \rightarrow 0} E(u, r)$ do so. Then we can find a number $\left.r_{0} \in\right] 0, \min \left\{1 / 4,\left(A^{\frac{3}{2}}(u, 1)+D_{0}^{2}(u, 1)\right)^{-2}\right\}[$ such that

$$
E\left(u, r_{0}\right)<\frac{3}{4} \varepsilon_{10}(m)
$$

and thus

$$
g_{r_{0}}(u, q)<\varepsilon_{10}(m) .
$$

By Proposition 3.1, the point $z=0$ is a regular point of $u$ and therefore it is a regular point of $v$. Proposition 3.2 is proved.

In the same way, one can prove the following statements.

Proposition 3.3 Let the pair $v$ and $p$ be a suitable weak solution to the $N S E$ 's in $Q$.

For any $M>0$, there exists a positive number $\varepsilon_{2}(M)$ such that if

$$
\limsup _{r \rightarrow 0} A(r)<M
$$

and

$$
g<\varepsilon_{2}(M)
$$

then $z=0$ is a regular point of $v$.

Proposition 3.4 Let the pair $v$ and $p$ be a suitable weak solution to the $N S E$ 's in $Q$.

For any $M>0$, there exists a positive number $\varepsilon_{3}(M)$ such that if

$$
\limsup _{r \rightarrow 0} C(r)<M
$$


and

$$
g<\varepsilon_{3}(M)
$$

then $z=0$ is a regular point of $v$.

Proof of Theorem 1.4 It is a direct consequence of Propositions 3.2 3.4. Theorem 1.4 is proved.

Let us give some comments on the proof of Theorem 1.7. The crucial point is an analog of Proposition 3.1. It can be formulated as follows.

Proposition 3.5 Let the pair $v$ and $p$ be a suitable weak solution to the $N S E$ 's in $Q$.

For any $M>0$, there exists a positive number $\widehat{\varepsilon}_{10}=\widehat{\varepsilon}_{10}(M)$ with the following property. If

$$
\sup _{0<r \leq 1} E(r)=E_{0} \leq M
$$

and

$$
E_{3}\left(r_{*}\right)<\widehat{\varepsilon}_{10}(M)
$$

for some $\left.r_{*} \in\right] 0, \min \left\{1 / 4,\left(A^{\frac{3}{2}}(1)+D_{0}^{2}(1)\right)^{-2}\right\}[$, then $z=0$ is a regular point of $v$.

Proof We repeat the proof of Proposition 3.1 with the following modifications. Instead of (3.4), we have

$$
E_{3}\left(v^{n}, r_{n}\right)=\frac{1}{r_{n}} \int_{Q\left(r_{n}\right)}\left|v_{, 3}^{n}\right|^{2} d z \leq \frac{1}{n} .
$$

Now, (3.10) should be replaced with

$$
E_{3}\left(u^{n_{k}}, 1\right) \rightarrow 0 \quad \text { as } \quad n \rightarrow+\infty
$$

The latter implies

$$
u_{, 3}^{n_{k}} \rightarrow 0 \quad \text { in } \quad L_{2}(Q)
$$

and thus $u_{, 3}=0$ in $Q$. This makes it possible to introduce a new function $U\left(x_{1}, x_{2}, t\right)=u\left(x_{1}, x_{2}, x_{3}, t\right)$ which satisfies the inequalities

$$
E(u, r) \geq \frac{1}{r} \int_{-r^{2}}^{0} d t \int_{-r / \sqrt{2}}^{r / \sqrt{2}} d x_{3} \int_{x_{1}^{2}+x_{2}^{2}<r^{2} / 2}\left|\nabla_{2} U\right|^{2} d x_{1} d x_{2}=
$$




$$
=\sqrt{2} \int_{-r^{2}}^{0} \int_{x_{1}^{2}+x_{2}^{2}<r^{2} / 2}\left|\nabla_{2} U\right|^{2} d x_{1} d x_{2} d t \geq \frac{1}{\sqrt{2}} E(u, r / \sqrt{2})
$$

for any $0<r \leq 1$. Here, $\nabla_{2}$ is the two-dimensional gradient.

So, we can state that

$$
\int_{-1}^{0} \int_{x_{1}^{2}+x_{2}^{2}<1 / 2}\left|\nabla_{2} U\right|^{2} d x_{1} d x_{2} d t \leq E(u, 1)<+\infty
$$

and thus

$$
\int_{-r^{2}}^{0} \int_{x_{1}^{2}+x_{2}^{2}<r^{2} / 2}\left|\nabla_{2} U\right|^{2} d x_{1} d x_{2} d t \rightarrow 0 \quad \text { as } \quad r \rightarrow 0 .
$$

This means that $E(r) \rightarrow 0$ as $r \rightarrow 0$ and, by Theorem $1.2, z=0$ is a regular point of $u$. So, we have shown the validity of (3.23) and (3.25).

Next, we proceed in a slightly different way. By (2.4), we have

$$
D_{0}\left(q^{n_{k}}, r\right) \leq c\left[\left(\frac{r}{\varrho}\right)^{\frac{5}{2}} D_{0}\left(q^{n_{k}}, \varrho\right)+\left(\frac{\varrho}{r}\right)^{2} C\left(u^{n_{k}}, \rho\right)\right]
$$

for all $0<r<\varrho \leq 1$. Thanks to (3.12), the new version of the previous inequality can be given. It has the form

$$
D_{0}\left(q^{n_{k}}, r\right) \leq c \widetilde{d}_{0}(M)\left[\left(\frac{r}{\varrho}\right)^{\frac{5}{2}}+\left(\frac{\varrho}{r}\right)^{2} C\left(u^{n_{k}}, \rho\right)\right]
$$

for all $0<r<\varrho \leq 1$. Passing to the limit as $k \rightarrow+\infty$, we find from (3.15) the estimate

$$
\limsup _{k \rightarrow 0} D_{0}\left(q^{n_{k}}, r\right) \leq c \widetilde{d}_{0}(M)\left[\left(\frac{r}{\varrho}\right)^{\frac{5}{2}}+\left(\frac{\varrho}{r}\right)^{2} C(u, \rho)\right]
$$

for all $0<r<\varrho \leq 1$. Setting $\varrho=\gamma r \leq r_{1}$, we derive from (3.23) the inequality

$$
\begin{gathered}
\limsup _{k \rightarrow 0} D_{0}\left(q^{n_{k}}, r\right) \leq c \widetilde{d}_{0}(M)\left[\gamma^{-\frac{5}{2}}+c \gamma^{2} d_{3}^{3}(M) \gamma^{3} r^{3}\right] \\
\leq c \widetilde{d}_{4}(M)\left[\gamma^{-\frac{5}{2}}+\gamma^{5} r^{3}\right]
\end{gathered}
$$


which is valid for any $\gamma>1$ and for any $r>0$ satisfying the condition $\gamma r \leq r_{1}$. So, we have (see (3.25) )

$$
c d_{3}^{3}(M) r^{3}+c \widetilde{d}_{4}(M) \gamma^{-\frac{5}{2}}+c \widetilde{d}_{4}(M) \gamma^{5} r^{3} \geq \varepsilon
$$

for the same $\gamma$ and $r$ as in the previous inequality. Let us choose $\gamma_{0}(\varepsilon, M)>1$ and fix it so that

$$
c \widetilde{d}_{4}(M) \gamma_{0}^{-\frac{5}{2}}(\varepsilon, M) \leq \varepsilon / 4
$$

Then

$$
c d_{3}^{3}(M) r^{3}+c \widetilde{d}_{4}(M) \gamma_{0}^{5}(\varepsilon, M) r^{3} \geq 3 \varepsilon / 4
$$

for all $0<r \leq r_{1} / \gamma_{0}(\varepsilon, M)$. The latter inequality does not hold for sufficiently small $r$. Proposition (3.5) is proved.

The remaining part of the proof of Theorem 1.7 is the same as in Theorem 1.4

\section{Appendix}

Consider the initial boundary value problem for the following system of linear equations

$$
\partial_{t} v+u \cdot \nabla v-\Delta v=f \quad \text { in } \quad Q .
$$

We assume that unknown vector-valued function $v: Q \rightarrow \mathbb{R}^{3}$ satisfies the homogeneous conditions on the parabolic boundary $\partial^{\prime} Q$, i.e.,

$$
\left.v\right|_{\partial^{\prime} Q}=0 \text {. }
$$

Here, $u: Q \rightarrow \mathbb{R}^{3}$ and $f: Q \rightarrow \mathbb{R}^{3}$ are given functions satisfying the following conditions

$$
u \in L_{2}(Q), \quad \operatorname{div} u=0 \quad \text { in } Q \text {, }
$$

and

$$
f \in L_{\frac{5}{3}}(Q)
$$

Definition 4.1 The function $v$ is called a weak solution to initial boundary value problem (4.1) and (4.2) if it satisfies the conditions:

$$
v \in L_{2, \infty}(Q) \cap L_{2}\left(-1,0 ; \stackrel{\circ}{W}{ }_{2}^{1}(B)\right)
$$




$$
\begin{gathered}
t \rightarrow \int_{B} v(x, t) \cdot w(x) d x \text { is continuous on }[-1,0] \text { for any } w \in L_{2}(B) ; \\
\qquad \int_{Q}\left(-v \cdot \partial_{t} w-v \otimes u: \nabla w+\nabla v: \nabla w\right) d z=\int_{Q} f \cdot w d z
\end{gathered}
$$

for any $w \in C_{0}^{\infty}(Q)$;

$$
\|v(\cdot, t)\|_{L_{2}(B)} \rightarrow 0
$$

as $t \rightarrow-1+0$;

$$
\frac{1}{2} \int_{B}|v(x, t)|^{2} d x+\int_{-1}^{t} \int_{B}|\nabla v|^{2} d x d t^{\prime} \leq \int_{-1}^{t} \int_{B} f \cdot v d x d t^{\prime}
$$

for all $t \in[-1,0]$.

Theorem 4.2 Assume that conditions (4.3) and (4.4) hold. There exists at least one weak solution $v$ to initial boundary value problem (4.1), 4.2). In addition, it has the following differentiability properties

$$
v \in L_{3, \infty}(Q) \cap L_{5}(Q) .
$$

Moreover, if

$$
u \in L_{\frac{10}{3}}(Q)
$$

then problem (4.1), (4.2) has a unique weak solution.

Proof We start with the proof of the existence. It is easy to find smooth functions $u^{\delta}$ and $f^{\delta}$ with the following properties

$$
\operatorname{div} u^{\delta}=0, \quad u^{\delta} \rightarrow u \quad \text { in } \quad L_{2}(Q)
$$

and

$$
f^{\delta} \rightarrow f \quad \text { in } \quad L_{\frac{5}{3}}(Q)
$$

as $\delta \rightarrow 0$. As it is shown in [6], see also 4], there is a unique smooth solution $v^{\delta}$ to the initial boundary value problem:

$$
\partial_{t} v^{\delta}+u^{\delta} \cdot \nabla v^{\delta}-\Delta v^{\delta}=f^{\delta} \quad \text { in } \quad Q
$$

and

$$
\left.v^{\delta}\right|_{\partial^{\prime} Q}=0
$$


Lemma 4.3 Assume that we are given three sufficiently smooth functions $v$, $u$, and $f$ satisfying (4.1) and (4.2). Then, for all $t \in]-1,0[$, the following inequalities are valid:

$$
\begin{gathered}
\partial_{t} \int_{B}|v|^{2} d x+\int_{B}|\nabla v|^{2} d x \leq c\left[\left(\int_{B}|f|^{\frac{5}{3}} d x\right)^{\frac{1}{2}}+\left(\int_{B}|f|^{\frac{5}{3}} d x\right.\right. \\
\left.\left.+\int_{B}|\operatorname{div} u|^{\frac{5}{2}} d x\right) \int_{B}|v|^{2} d x\right]
\end{gathered}
$$

and

$$
\begin{gathered}
\partial_{t}|w|^{2} d x+\int_{B}|\nabla w|^{2} d x \leq c\left[\int_{B}|f|^{\frac{5}{3}} d x\left(\int_{B}|w|^{2} d x\right)^{\frac{4}{9}}\right. \\
\left.+\int_{B}|\operatorname{div} u|^{\frac{5}{2}} d x \int_{B}|w|^{2} d x\right]
\end{gathered}
$$

where $w=|v|^{\frac{3}{2}}$.

Proof Multiplying identity (4.1) by $v$ and integrating by parts, we have

$$
\frac{1}{2} \partial_{t} \int_{B}|v|^{2} d x+\int_{B}|\nabla v|^{2} d x=\int_{B} f \cdot v d x+\frac{1}{2} \int_{B}|v|^{2} \operatorname{div} u d x=I_{1}+I_{2} .
$$

Now, our aim is to estimates $I_{1}$ and $I_{2}$. For $I_{1}$, we use Hölder's inequality

$$
I_{1} \leq\left(\int_{B}|f|^{\frac{5}{3}} d x\right)^{\frac{3}{5}}\left(\int_{B}|v|^{\frac{5}{2}} d x\right)^{\frac{2}{5}} .
$$

The second multiplier on the right hand side of the latter relation can be estimated with the help of the multiplicative inequality

$$
I_{1} \leq c\left(\int_{B}|f|^{\frac{5}{3}} d x\right)^{\frac{3}{5}}\left(\int_{B}|v|^{2} d x\right)^{\frac{7}{20}}\left(\int_{B}|\nabla v|^{2} d x\right)^{\frac{3}{20}} .
$$

Next, by Young's inequality,

$$
I_{1} \leq \frac{1}{4} \int_{B}|\nabla v|^{2} d x+c\left(\int_{B}|f|^{\frac{5}{3}} d x\right)^{\frac{12}{17}}\left(\int_{B}|v|^{2} d x\right)^{\frac{7}{17}} .
$$


Since $5 / 17+7 / 17=12 / 17$, one may apply Young's inequality once more. As a result, we have

$$
I_{1} \leq \frac{1}{4} \int_{B}|\nabla v|^{2} d x+c \int_{B}|f|^{\frac{5}{3}} d x \int_{B}|v|^{2} d x+c\left(\int_{B}|f|^{\frac{5}{3}} d x\right)^{\frac{1}{2}} .
$$

Now, we are going to evaluate $I_{2}$. Hölder's inequality gives

$$
I_{2} \leq \frac{1}{2}\left(\int_{B}|v|^{\frac{10}{3}} d x\right)^{\frac{3}{5}}\left(\int_{B}|\operatorname{div} u|^{\frac{5}{2}} d x\right)^{\frac{2}{5}} .
$$

Then we use a multiplicative inequality

$$
I_{2} \leq c\left(\int_{B}|\nabla v|^{2} d x\right)^{\frac{3}{5}}\left(\int_{B}|v|^{2} d x\right)^{\frac{2}{5}}\left(\int_{B}|\operatorname{div} u|^{\frac{5}{2}} d x\right)^{\frac{2}{5}} .
$$

And, by Young's inequality,

$$
I_{2} \leq \frac{1}{4} \int_{B}|\nabla v|^{2} d x+c \int_{B}|\operatorname{div} u|^{\frac{5}{2}} d x \int_{B}|v|^{2} d x .
$$

Now, (4.16) follows from (4.18)-(4.20).

To prove the second estimate, we proceed as follows. First, we multiply identity (4.1) by $|v| v$ and then integrate by parts. As a result, we have the identity

$$
\begin{gathered}
\frac{1}{3} \partial_{t} \int_{B}|v|^{3} d x+\int_{B}|v|\left(|\nabla v|^{2}+|\nabla| v||^{2}\right) d x=\int_{B}|v| f \cdot v d x \\
+\frac{1}{3} \int_{B}|v|^{3} \operatorname{div} u d x,
\end{gathered}
$$

which implies the inequality

$$
\frac{1}{3} \partial_{t} \int_{B}|w|^{2} d x+\frac{4}{9} \int_{B}|\nabla w|^{2} d x \leq J_{1}+J_{2},
$$

where

$$
J_{1}=\left(\int_{B}|f|^{\frac{5}{3}} d x\right)^{\frac{3}{5}}\left(\int_{B}|w|^{\frac{10}{3}} d x\right)^{\frac{2}{5}}, \quad J_{2}=\frac{1}{3} \int_{B}|w|^{2} \operatorname{div} u d x
$$


The term $J_{2}$ is estimated in the same way as $I_{2}$. So,

$$
J_{2} \leq \frac{1}{18} \int_{B}|\nabla w|^{2} d x+c \int_{B}|\operatorname{div} u|^{\frac{5}{2}} d x \int_{B}|w|^{2} d x .
$$

For $J_{1}$, one can apply a multiplicative inequality and Young's inequality and find

$$
\begin{aligned}
J_{1} & \leq c\left(\int_{B}|f|^{\frac{5}{3}} d x\right)^{\frac{3}{5}}\left(\int_{B}|w|^{2} d x\right)^{\frac{4}{15}}\left(\int_{B}|\nabla w|^{2} d x\right)^{\frac{2}{5}} \\
& \leq \frac{1}{18} \int_{B}|\nabla w|^{2} d x+\int_{B}|f|^{\frac{5}{3}} d x\left(\int_{B}|w|^{2} d x\right)^{\frac{4}{9}} .
\end{aligned}
$$

Combining the latter estimate with (4.21) and (4.22), we complete the proof of (4.18). Lemma 4.3 is proved.

Now, we proceed with the proof of the theorem. From Lemma 4.3 and from Gronwall's lemma, it follows that certain norms of $v^{\delta}$ and $w^{\delta}=\left|v^{\delta}\right|^{\frac{3}{2}}$ are bounded uniformly with respect to $\delta$ :

$$
\left\|v^{\delta}\right\|_{L_{3, \infty}(Q)}+\left\|w^{\delta}\right\|_{L_{2, \infty}(Q)}+\left\|\nabla v^{\delta}\right\|_{L_{2}(Q)}+\left\|\nabla w^{\delta}\right\|_{L_{2}(Q)} \leq c<+\infty .
$$

Moreover, by a multiplicative inequality and by (4.23),

$$
\left\|v^{\delta}\right\|_{L_{5}(Q)} \leq c<+\infty
$$

and thus

$$
\left\|\left|v^{\delta}\left\|u^{\delta} \mid\right\|_{L_{10 / 7}(Q)} \leq c<+\infty .\right.\right.
$$

The latter, together with identity (4.7), allows us to state that

$$
\left\|\partial_{t} v^{\delta}\right\|_{L_{10 / 7}\left(-1,0 ; W_{10 / 7}^{-1}(B)\right)} \leq c<+\infty .
$$

Now, the existence of at least one weak solution to initial boundary value problem (4.1) and (4.2) can be deduced from estimates (4.23)-(4.26) in a more or less standard way.

Now, let us switch to the proof of the uniqueness of weak solutions to initial boundary value problem (4.1) and (4.2) in the class of weak solutions in the sense of Definition 4.1. We start with a simple remark

$$
\stackrel{\circ}{W}_{2}^{1}(Q) \cap L_{5}(Q)=\left[C_{0}^{\infty}(Q)\right]_{W_{2}^{1}(Q) \cap L_{5}(Q)} .
$$


Assume that $v^{1}$ is another solution to initial boundary value problem (4.1) and (4.2). By (4.11),

$$
u \cdot \nabla v^{1} \in L_{\frac{5}{4}}(Q) .
$$

Taking into account (4.27) and (4.28), let us transform identity (4.7) into the form:

$$
\int_{Q}\left(-v^{1} \cdot \partial_{t} w+\left(u \cdot \nabla v^{1}\right) \cdot w+\nabla v: \nabla w\right) d z=\int_{Q} f \cdot w d z
$$

for any $w \in \stackrel{\circ}{W} \underset{2}{1}(Q) \cap L_{5}(Q)$.

On the other hand, according to the first part of the theorem, we have

$$
|v||u| \in L_{2}(Q)
$$

and thus

$$
\int_{Q}\left(-v \cdot \partial_{t} w-v \otimes u: \nabla w+\nabla v: \nabla w\right) d z=\int_{Q} f \cdot w d z
$$

for any $w \in \stackrel{\circ}{W_{2}^{1}}(Q)$.

Fix an arbitrary number $\varepsilon \in] 0,1 / 4[$ and take a nonnegative smooth cutoff function $\chi$ having a support in $]-1+\varepsilon / 2,-\varepsilon / 2\left[\right.$. Let $\omega_{\varrho}$ be a standard smoothing kernel and let

$$
v_{\varrho}(x, t)=\int_{-1}^{0} \omega_{\varrho}(t-s) v(x, s) d s
$$

Now, we introduce two test functions:

$$
w=\left(\chi v_{\varrho}\right)_{\varrho}, \quad w^{1}=\left(\chi v_{\varrho}^{1}\right)_{\varrho} .
$$

For sufficiently small $\varrho$, we have

$$
w \in \stackrel{\circ}{W}_{2}^{1}(Q) \cap L_{5}(Q), \quad w^{1} \in \stackrel{\circ}{W}_{2}^{1}(Q) .
$$

Next, we use the first test function in (4.30) and then both of them in (4.31). Taking into account well-known properties of smoothing kernel, we can produce three identities: 


$$
\begin{aligned}
\int_{Q}\left(-v_{\varrho}^{1} \cdot \partial_{t}\left(\chi v_{\varrho}\right)+\right. & \left.\left(u \cdot \nabla v^{1}\right)_{\varrho} \cdot \chi v_{\varrho}+\nabla v_{\varrho}^{1}: \chi \nabla v_{\varrho}\right) d z \\
& =\int_{Q} f_{\varrho} \cdot v_{\varrho} d z \\
\int_{Q}\left(-v_{\varrho} \cdot \partial_{t}\left(\chi v_{\varrho}^{1}\right)-\right. & \left.(v \otimes u)_{\varrho}: \chi \nabla v_{\varrho}^{1}+\nabla v_{\varrho}: \chi \nabla v_{\varrho}^{1}\right) d z \\
& =\int_{Q} f_{\varrho} \cdot v_{\varrho}^{1} d z,
\end{aligned}
$$

and

$$
\begin{aligned}
\int_{Q}\left(-v_{\varrho} \cdot \partial_{t}\left(\chi v_{\varrho}\right)-\right. & \left.(v \otimes u)_{\varrho}: \chi \nabla v_{\varrho}+\nabla v_{\varrho}: \chi \nabla v_{\varrho}\right) d z \\
= & \int_{Q} f_{\varrho} \cdot v_{\varrho} d z .
\end{aligned}
$$

Add (4.33) and (4.34) and integrate the sum by parts, we find

$$
\begin{gathered}
\int_{Q}\left(-v_{\varrho} \cdot v_{\varrho}^{1} \partial_{t} \chi+\left(u \cdot \nabla v^{1}\right)_{\varrho} \cdot \chi v_{\varrho}-(v \otimes u)_{\varrho}: \chi \nabla v_{\varrho}^{1}\right. \\
\left.\quad+2 \chi \nabla v_{\varrho}: \nabla v_{\varrho}^{1}\right) d z=\int_{Q} f_{\varrho} \cdot\left(v_{\varrho}+v_{\varrho}^{1}\right) d z .
\end{gathered}
$$

Passing to the limit as $\varrho \rightarrow 0$, we arrive at the identity

$$
\int_{Q}\left(-v \cdot v^{1} \partial_{t} \chi+2 \chi \nabla v: \nabla v^{1}\right) d z=\int_{Q} f \cdot\left(v+v^{1}\right) d z .
$$

Now, we argue as follows. Taking into account two properties of weak solutions (4.6) and (4.8) and choosing our cut-off function $\chi$ in an appropriate way, we can state that

$$
\int_{B} v(x, t) \cdot v^{1}(x, t) d x+2 \int_{-1}^{t} \int_{B} \nabla v: \nabla v^{1} d x d t^{\prime}=\int_{-1}^{t} \int_{B} f \cdot\left(v+v^{1}\right) d x d t^{\prime}
$$


for a.a. $t \in]-1,0[$. Proceeding in the same way, we derive from (4.35) another identity

$$
\frac{1}{2} \int_{B}|v(x, t)|^{2} d x+\int_{-1}^{t} \int_{B}|\nabla v|^{2} d x d t^{\prime}=\int_{-1}^{t} \int_{B} f \cdot v d x d t^{\prime}
$$

for a.a. $t \in]-1,0\left[\right.$. But $v^{1}$ is a weak solution and therefore it obeys global energy inequality (4.9)

$$
\frac{1}{2} \int_{B}\left|v^{1}(x, t)\right|^{2} d x+\int_{-1}^{t} \int_{B}\left|\nabla v^{1}\right|^{2} d x d t^{\prime} \leq \int_{-1}^{t} \int_{B} f \cdot v^{1} d x d t^{\prime}
$$

for all $t \in[-1,0]$. From (4.37)-(4.39), it is easy to derive the estimate

$$
\frac{1}{2} \int_{B}\left|v^{1}(x, t)-v(x, t)\right|^{2} d x+\int_{-1}^{t} \int_{B}\left|\nabla\left(v^{1}-v\right)\right|^{2} d x d t^{\prime} \leq 0
$$

for a.a. $t \in]-1,0\left[\right.$. The latter implies $v^{1}=v$. Theorem (4.2) is proved.

Now, let us consider the pair $v$ and $p$ being a suitable weak solution to the Navier-Stokes equations in $Q$, see definition 1.1. Our aim is

Proposition 4.4 Assume that the pair $v$ and $p$ is a suitable weak solution in $Q$. Suppose that $p$ is independent of spatial variables $x$. Then $v$ is Hölder continuous in the $\bar{Q}(3 / 8)$.

Proof Fix a smooth cut-off function $\psi$ vanishing in a neighborhood of $\partial^{\prime} Q$ and being equal to one in $\bar{Q}(3 / 4)$. Let

$$
\bar{v}=\psi v, \quad f=v \partial_{t} \psi-2 \nabla v \nabla \psi-v \Delta \psi+v \cdot \nabla \psi v, \quad u=v
$$

According to the theory of multiplicative inequalities,

$$
u \in L_{\frac{10}{3}}(Q), \quad \operatorname{div} u=0 .
$$

Moreover,

$$
\bar{v} \in L_{2, \infty}(Q) \cap L_{2}\left(-1,0 ; \stackrel{\circ}{W}_{2}^{1}(B)\right)
$$


and

$$
\int_{Q}\left(-\bar{v} \cdot \partial_{t} w-\bar{v} \otimes u: \nabla w+\nabla \bar{v}: \nabla w\right) d z=\int_{Q} f \cdot w d z
$$

for any $w \in C_{0}^{\infty}(Q)$.

Now, let us prove that $\bar{v}$ is a weak solution to the initial boundary value problem (4.1) and (4.2). By (4.41) and (4.42), it remains to verify that $\bar{v}$ satisfies (4.6) and (4.9). Obviously, (4.8) is fulfilled since $\bar{v}$ is identically equal to zero in a neighborhood of $t=0$.

To check (4.6), we note that

$$
\bar{v} \otimes u \in L_{\frac{5}{3}}(Q) .
$$

This, together with (4.42), gives us the estimate for

$$
\left\|\partial_{t} \bar{v}\right\|_{L_{5 / 3}\left(-1,0 ; W_{5 / 3}^{-1}(B)\right)}<+\infty .
$$

Then, (4.6) follows from (4.41).

Next, integrating by parts, we can derive the following identity

$$
\begin{aligned}
I(t)= & \int_{B}|\bar{v}(x, t)|^{2} d x+2 \int_{-1}^{t} \int_{B}|\nabla \bar{v}|^{2} d x d t^{\prime}-2 \int_{-1}^{t} \int_{B} f \cdot \bar{v} d x d t^{\prime} \\
& =\int_{B} \psi^{2}(x, t)|v(x, t)|^{2} d x+2 \int_{-1}^{t} \int_{B} \psi^{2}|\nabla v|^{2} d x d t^{\prime} \\
& -\int_{-1}^{t} \int_{B}\left(|v|^{2}\left(\Delta \psi^{2}+\partial_{t} \psi^{2}\right)+v \cdot \nabla \psi^{2}|v|^{2}\right) d x d t^{\prime} .
\end{aligned}
$$

By assumptions of Proposition 4.4, $I(t) \leq 0$ for a.a. $t \in]-1,0[$. Here, we have used the identity

$$
\int_{B} v \cdot \nabla \psi^{2} p d x=0
$$

However, (4.6) means that the inequality $I(t) \leq 0$ holds for all $t \in[-1,0]$. So, we have demonstrated that $\bar{v}$ is a weak solution to initial boundary value problem (4.1) and (4.2). Then, according to Theorem 4.2, we can state that

$$
\bar{v} \in L_{3, \infty}(Q)
$$


and thus

$$
v \in L_{3, \infty}(Q(3 / 4)) .
$$

In this case, we have a suitable weak solution $v$ and $p$ in $Q(3 / 4)$ which satisfies additional condition (4.43). Using scaling and results of [3], we show that $v$ is Hölder continuous in the closure of the set $Q(3 / 8)$. Proposition 4.4 is proved.

\section{References}

[1] Caffarelli, L., Kohn, R.-V., Nirenberg, L., Partial regularity of suitable weak solutions of the Navier-Stokes equations, Comm. Pure Appl. Math., Vol. XXXV (1982), pp. 771-831.

[2] Choe, H. L., Lewis, J. L., On the singular set in the Navier-Stokes equations, J. Functional Anal., 175(2000), pp. 348-369.

[3] Escauriaza,L., Seregin, G., Šverák, V.,. $L_{3, \infty}$-Solutions to the NavierStokes equations and backward uniqueness, Uspekhi Matematicheskih Nauk, v. 58, 2(350), pp. 3-44. English translation in Russian Mathematical Surveys, 58(2003)2, pp. 211-250.

[4] Kiselev, A. A., Ladyzheskaya O. A. On exitence and uniqueness of the solution of the nonstationary problem for a viscous incompressible fluid, Izvestiya Akad. Nauk SSSR 21(1957), 655-680.

[5] Ladyzhenskaya, O. A., Seregin, G. A., On partial regularity of suitable weak solutions to the three-dimensional Navier-Stokes equations, J. math. fluid mech., 1(1999), pp. 356-387.

[6] Ladyzhenskaya, O. A., Solonnikov, V. A., Uralt'seva, N. N., Linear and quasi-linear equations of parabolic type, Moscow, 1967; English translation, American Math. Soc., Providence 1968.

[7] Leray, J., Sur le mouvement d'un liquide visqueux emplissant l'espace, Acta Math. 63(1934), pp. 193-248.

[8] Lin, F.-H., A new proof of the Caffarelly-Kohn-Nirenberg theorem, Comm. Pure Appl. Math., 51(1998), no.3, pp. 241-257. 
[9] Seregin, G.A., On smoothness of $L_{3, \infty}$-solutions to the Navier-Stokes equations up to boundary, Mathematische Annalen, 332(2005), pp. 219238.

[10] Seregin, G.A., Local Regularity Theory of the Navier-Stokes equations, to appear in Handbook of Mathematical Fluid Mechanics, Vol. 4.

[11] Seregin, G.A., Estimates of suitable weak solutions to the Navier-Stokes equations in critical Morrey spaces, to appear in Zapiski Nauchnuh Seminarov, POMI.

[12] Seregin, G., Šverák, V., The Navier-Stokes equations and backward uniqueness, Nonlinear Problems in Mathematical Physics II, In Honor of Professor O.A. Ladyzhenskaya, International Mathematical Series II, 2002, pp. 359-370.

[13] Serrin, J., On the interior regularity of weak solutions of the NavierStokes equations, Arch. Ration. Mech. Anal., 9(1962), pp. 187-195.

[14] Struwe, M., On partial regularity results for the Navier-Stokes equations, Comm. Pure Appl. Math., 41 (1988), no. 4, 437-458.

G. Seregin

Steklov Institute of Mathematics at St.Petersburg,

Fontanka 27, 191023 St.Peterburg, Russia,

seregin@pdmi.ras.ru 